\title{
Intact Behavioral Expression of Contextual Fear, Context Discrimination, and Object Discrimination Memories Acquired in the Absence of the Hippocampus
}

\author{
Darryl C. Gidyk, ${ }^{1,2}$ Robert J. McDonald, ${ }^{1}$ and Robert J. Sutherland ${ }^{1}$ \\ ${ }^{1}$ Department of Neuroscience, Canadian Centre for Behavioural Neuroscience, The University of Lethbridge, Lethbridge, Alberta T1K 3M4, Canada, \\ and ${ }^{2}$ Sunnybrook Research Institute, Sunnybrook Health Sciences Centre, Toronto, Ontario M4N 3M5, Canada
}

We test the hypothesis that the stability and precision of context and visual discrimination memories depend on interactions between the hippocampus (HPC) and other memory storage networks. In four experiments we tested the properties of memories acquired in the absence of the HPC. Long-Evans male rats were exclusively used in all experiments. Experiment 1 evaluated acquisition and retention of context fear memories in rats with prior partial or complete HPC damage. Confirming an earlier report (Zelikowsky et al., 2012) a very small but statistically reliable slowing in a single session of context fear conditioning was found after HPC damage. In contrast, retention of context fear memory was normal after HPC damage up to $30 \mathrm{~d}$ after learning. In experiment 2 , we found that discrimination between a context paired with foot shocks and a different context never paired with foot shock was retained normally for $15 \mathrm{~d}$. In experiment 3 , we replicated the finding of intact context discrimination for at least $15 \mathrm{~d}$ in rats who display a significant impairment in acquisition of place learning in the Morris water task (MWT). In final experiment using an appetitive object discrimination task, we showed normal retention of the discrimination for at least $30 \mathrm{~d}$ after training in rats with complete HPC damage. These finding score against the idea that non HPC memory storage requires a period of interaction with HPC to establish a stable, precise memory.

Key words: consolidation; discrimination; hippocampus; long-term memory

Significance Statement

Contrary to expectations from systems memory consolidation, we find that in the absence of a functional hippocampus (HPC) context and visual memories are formed rapidly and exhibit normal persistence and precision. The findings suggest that the HPC is not obligatory for these features of long-term memories.

\section{Introduction}

The longevity and precision of long-term memories are hypothesized to depend on the hippocampus (HPC) and its role in a systems consolidation process. Systems consolidation is a hypothetical process by which long-term memories gradually transition from being hippocampal dependent to hippocampal independent; such memories become more stable but lose many details and precision (Wiltgen and Tanaka, 2013; Sekeres et al., 2017). In partial support of this idea, damage to the rodent HPC after learning results in retrograde amnesia for contextual fear memory, context discriminations, and visual discriminations (Kim and Fanselow, 1992; Maren et al., 1997;

\footnotetext{
Received Mar. 6, 2020; revised May 7, 2020; accepted June 21, 2020.

Author contributions: D.C.G., R.J.M., and R.J.S. designed research; D.C.G. performed research; D.C.G. analyzed data; D.C.G., R.J.M., and R.J.S. wrote the paper.

The authors declare no competing financial interests.

This work was supported by the Natural Sciences and Engineering Research Council of Canada (R.J.S.).

Correspondence should be addressed to Darryl C. Gidyk at darryl.gidyk@sri.utoronto.ca.

https://doi.org/10.1523/JNEUROSCI.0546-20.2020

Copyright $\odot 2021$ the authors
}

Anagnostaras et al., 1999; Mumby et al., 1999; Sutherland et al., 2001; Driscoll et al., 2005; Lehmann et al., 2007, 2013; Epp et al., 2008; Broadbent and Clark, 2013; Sparks et al., 2013; Lee et al., 2017). Some data suggest that long-term memories rapidly decay when created in the absence of an intact HPC. One study found that contextual fear memory was unaffected by prelearning damage to the dorsal HPC (dHPC) $24 \mathrm{~h}$ after learning, but was impaired when rats were tested 3,10 , or $30 \mathrm{~d}$ later (Zelikowsky et al., 2012). dHPC, HPC, and fornix-damaged rats exhibit minor to severe memory decay on two-choice visual discriminations (Vnek and Rothblat, 1996; Wiig et al., 1996; Broadbent et al., 2007). Prelearning HPC damage has also been reported to impair context discrimination (Frankland et al., 1998; Antoniadis and McDonald, 2000). However, HPC damage before learning does not always cause anterograde amnesia or impair discriminative behaviors (Wiltgen et al., 2006; Epp et al., 2008; Lee et al., 2016). Taken together, the question of whether non-HPC networks can form, maintain, and express stable long-term memories, including those with sufficient detail to support discriminations remains unanswered. 
Studies that damaged HPC before learning and found memory impairments have spared some unknown degree of hippocampal function (Wible et al., 1992; Vnek and Rothblat, 1996; Frankland et al., 1998; Zelikowsky et al., 2012). Partial hippocampal damage is known to have heterogenous effects on contextual fear memories. When damage is incomplete (e.g., $\leq 50 \%$ ), as if the dHPC is targeted and ventral HPC (vHPC) is spared, memory can remain partially intact (Scott et al., 2016), but not necessarily in a reliable pattern (Kim and Fanselow, 1992; Anagnostaras et al., 1999; Lehmann et al., 2007; Sutherland et al., 2008). Moreover, the vHPC is reciprocally connected to the medial prefrontal cortex, striatum, and amygdala, which collectively contribute to learning and memory (McDonald et al., 2007; Gruber and McDonald, 2012; McDonald and Hong, 2013; Kitamura et al., 2017). Therefore, it is unclear whether impairments after partial HPC damage can be attributed to a lack of persistence and detail in memory supported by non-hippocampal networks, or whether incomplete damage resulted in the acquisition of dysfunctional hippocampal memory, which could not be maintained and expressed normally. These questions are relevant to the wide range of clinical disorders involving HPC dysfunction as well. The HPC is especially vulnerable to the effects of aging (e.g., sporadic Alzheimer's disease), which causes deleterious effects on cognition in a heterogeneous manner (Gidyk et al., 2015). Uncovering compensatory mechanisms in memory and cognition may provide a key for future diagnosis and treatment of disorders related to HPC dysfunction and disease.

The present set of experiments tested the hypothesis that the HPC is necessary for the longevity and precision of long-term memories. Rats with or without damage to the HPC (or dHPC in experiment 1) underwent contextual fear conditioning, or training on a two-choice visual discrimination, then memory of independent groups of rats was tested at different retention intervals (context memory, 1, 3, 10, $30 \mathrm{~d}$; context discrimination, 1, 7, $15 \mathrm{~d}$; visual discrimination, $3,10,30 \mathrm{~d}$ ). The predictions of the standard model of systems consolidation include more rapid decay of memories and more rapid loss of memory precision. To ensure extensive damage to the HPC disrupted its function, a separate experiment tested rats on the spatial cue version of the Morris water task (MWT) before contextual fear conditioning and remote context discrimination testing. HPC damage was systematically quantified and reported for all experiments.

\section{Materials and Methods \\ Subjects}

Two hundred and five male Long-Evans hooded rats (Charles River), weighing 330-500 g at the time of surgery, were housed in pairs in standard cages (Allentown) in the University of Lethbridge rodent vivarium $\left(20^{\circ} \mathrm{C}, 50 \%\right.$ relative humidity). Access to food and water was ad libitum on arrival until endpoint except as described below (experiment 4). The vivarium light/dark cycle was $12 \mathrm{~h}$, with lights on at 07:30 A.M. daily. Rats were handled for several minutes daily during the week before surgery. All procedures conformed to guidelines set by the Canadian Council on Animal Care and were approved by the University of Lethbridge Animal Welfare Committee.

\section{dHPC lesion procedures}

Surgical procedures followed those reported by Zelikowsky et al. (2012). Rats were anesthetized with sodium pentobarbital $(65 \mathrm{mg} / \mathrm{kg}$, i.p.) and received atropine sulfate $(0.4 \mathrm{mg} / \mathrm{kg}$, i.p.) and ketoprofen $(1 \mathrm{mg} / \mathrm{kg}$, s.c.). The head was shaved, eye ointment was applied, and the scalp cleaned with chlorohexidine then $70 \%$ alcohol. Once fixed in the stereotaxic frame (David Kopf), a midline incision was made and the scalp retracted. Four small holes were drilled into the skull. Thirty-gauge stainless steel
Table 1. NMDA Infusion volumes and site coordinates

\begin{tabular}{cccccc}
\hline & Site & $\mathrm{A} / \mathrm{P}$ & $\mathrm{M} / \mathrm{L}$ & $\mathrm{D} / \mathrm{V}$ & Volume \\
\hline $\mathrm{dHPC}$ & 1 & -2.8 & \pm 1.6 & -3.5 & $0.4 \mu \mathrm{L}$ \\
& 2 & -4.2 & \pm 2.6 & -3.5 & $0.4 \mu \mathrm{L}$ \\
$\mathrm{HPC}$ & 1 & -2.6 & \pm 1.5 & -3.4 & $0.3 \mu \mathrm{L}$ \\
& 2 & -3.1 & \pm 1.5 & -3.5 & $0.4 \mu \mathrm{L}$ \\
& 3 & -4.1 & \pm 3.0 & -3.5 & $0.4 \mu \mathrm{L}$ \\
& 4 & -5.0 & \pm 3.0 & -3.5 & $0.4 \mu \mathrm{L}$ \\
& 5 & -5.0 & \pm 5.2 & -7.3 & $0.4 \mu \mathrm{L}$ \\
& 6 & -5.0 & \pm 4.4 & -4.4 & $0.4 \mu \mathrm{L}$ \\
& 7 & -5.8 & \pm 5.1 & -7.5 & $0.5 \mu \mathrm{L}$ \\
& 8 & -5.8 & \pm 5.1 & -6.0 & $0.5 \mu \mathrm{L}$ \\
\hline
\end{tabular}

dHPC coordinates and volumes from Zelikowsky et al. (2012); HPC coordinates and volumes adapted from Sparks et al. (2013). All coordinates expressed in millimeters relative to bregma.

injection cannulae, soldered into 23-gauge cannulae, were connected to $10-\mu \mathrm{l}$ micro-syringes (Hamilton Co) via polyethylene tubing (PE50) and attached to the arms of the stereotax. Syringes were mounted on an infusion pump (Harvard Apparatus) and NMDA $(20 \mathrm{mg} / \mathrm{ml}$; SigmaAldrich), dissolved in $0.01 \mathrm{M}$ PBS was drawn up into the cannulae and tubing. Infusions of NMDA were delivered sequentially, two at a time at corresponding bilateral sites $(0.15 \mu \mathrm{l} / \mathrm{min}$; coordinates and volumes; Table 1). Cannulae were left in place for $2 \mathrm{~min}$ following each bilateral infusion. Following the final infusion, the scalp incision was sutured and animals were placed in cages with soft paper bedding until fully recovered from anesthesia. The home cage surgical recovery period was $12 \mathrm{~d}$. Identical procedures were employed for SHAM surgeries, except the cannulae were not lowered into the brain and NMDA infusions were omitted.

\section{HPC lesion procedures}

Surgical procedures were adapted from previous work (Sparks et al., 2013; Lee et al., 2017). Rats were medicated with phenobarbital (30 mg/ $\mathrm{kg}$, i.p.) and ketoprofen ( $1 \mathrm{mg} / \mathrm{kg}$, s.c.), then anesthetized via isoflurane inhalation, beginning with $4 \%$ in $4 \mathrm{l} / \mathrm{min}$ oxygen for induction, then $1-$ $2 \%$ in $11 /$ min oxygen to maintain a surgical plane. Following surgical site preparation and scalp incision, 14 holes $(0.5-\mathrm{mm}$ diameter) were drilled in the skull and infusions were delivered in sequence $(7.5 \mathrm{mg} / \mathrm{ml}$ NMDA; $0.15 \mu \mathrm{l} / \mathrm{min}$; coordinates and volumes; Table 1$)$. The infusion cannulae were left in place for $3 \mathrm{~min}$ following delivery of NMDA. After all infusions, the scalp was sutured and diazepam $(5 \mathrm{mg} / \mathrm{kg}$, i. p.) was administered for seizure prophylaxis and repeated if needed. SHAM surgeries followed the same procedures, except the cannulae were not lowered, NMDA infusions were omitted, and diazepam was not administered. The surgical recovery period was $12 \mathrm{~d}$ (experiments 1,2,4), or $7 \mathrm{~d}$ (experiment 3 ).

\section{Shock context}

Contextual fear conditioning episodes and memory retention tests took place in a chamber with aluminum side walls and transparent acrylic rear wall, ceiling, and door (MED-Associates; outer dimensions: $33 \times 25$ $\times 27 \mathrm{~cm}$ ). The floor consisted of 19 stainless steel rods (4-mm diameter) spaced $1.3 \mathrm{~cm}$ apart, wired to a shock source and grid scrambler (MEDAssociates). The chambers were mounted in cabinets located in a standard testing room. Ventilation fans within the cabinet supplied background noise $(\sim 65 \mathrm{~dB})$. The chambers were cleaned thoroughly with dilute, germicidal quat-sanitizer $\left(4 \mathrm{ml} / \mathrm{l} \mathrm{H}_{2} \mathrm{O}\right.$; Quatsyl-D Plus, Pfizer Canada Inc.) between every session.

\section{Novel context}

A second chamber in a different testing room served as the novel context for discrimination tests. The chamber was a triangular prism with nontransparent black acrylic walls $(64 \times 64 \times 64 \mathrm{~cm})$ and a removable white acrylic lid (floor-to-ceiling height: $30 \mathrm{~cm}$ ). The grid floor made of stainless-steel bars was covered with a smooth transparent acrylic insert. The apparatus was cleaned before and between sessions with a dilute persulfate solution (Virkon; Vetoquinol N.A. Inc.). 


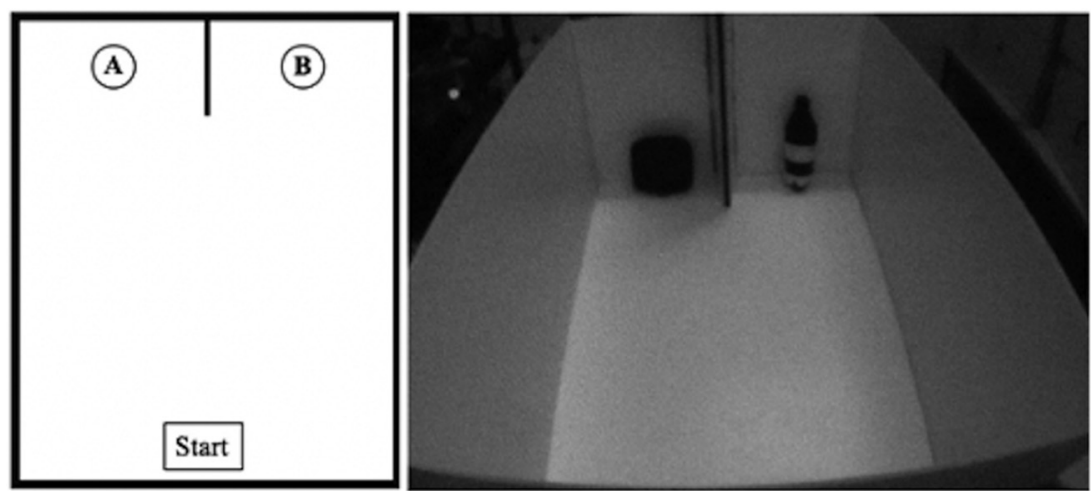

Figure 1. Schematic and still-frame capture of the dry land visual discrimination task arena. Left, Line drawing of the dry land visual discrimination arena, with reward dishes $A$ and $B$ and start position labeled. Right, Picture of the arena with the objects balanced on dishes before a trial (rectangle on $A$; cylinder on $B$ ).

\section{MWT}

All sessions were conducted in a circular fiberglass pool (125 cm dia) situated in a testing room equipped with a ceiling-mounted video camera connected to a laptop PC running HVS 2100 tracking software (HVS Image Ltd). The pool was filled with water $\left(21^{\circ} \mathrm{C}\right)$ and made opaque with non-toxic white paint before each session. The escape platform (13$\mathrm{cm}$ diameter) was located $\sim 2 \mathrm{~cm}$ below the surface of the water in the center of the North-West quadrant for all training sessions. Large posters of geometric shapes fixed to the walls of the testing room provided extra-maze visual cues that rats could use to navigate and learn the location of the platform. The pool was drained and thoroughly cleaned following completion of each session.

\section{Visual discrimination apparatus}

Pretraining, training, and testing took place in a custom-designed arena constructed from corrugated white plastic, with inner dimensions measuring; $90 \times 60 \times 45 \mathrm{~cm}$. The two-layered floor consisted of a removable insert resting atop the arena ultrastructure. The removable insert had two circular depressions cut into it, which secured reward dishes (2.54$\mathrm{cm}$ diameter) in place during trials, and allowed the dishes to be removed and cleaned between trials. The dishes were designed so objects could be placed on them by the experimenter before trials and displaced by rats during trials. The back wall of the box housed plastic tubes $(2-\mathrm{cm}$ diameter) positioned for manual delivery of reward to the dishes. A divider $(15 \mathrm{~cm})$ protruded from the center point of the back wall at a $90^{\circ}$ angle to deter rats from displacing both objects simultaneously (Fig. 1). The arena and its features were adapted from various sources (Mumby et al., 1999; Broadbent et al., 2007; Epp et al., 2008).

\section{Histology}

All animals received an overdose of sodium pentobarbital, then were perfused transcardially with $0.9 \%$ PBS, followed by $4 \%$ paraformaldehyde (PFA) in $0.9 \% 0.01$ м PBS. The brains were extracted and postfixed for at least $24 \mathrm{~h}$ in PFA-PBS solution, then transferred to 30\% sucrose in $0.9 \% 0.01 \mathrm{M}$ PBS with $0.02 \%$ sodium azide for at least $48 \mathrm{~h}$. The cryoprotected brains were frozen and sectioned in the coronal plane at $40 \mu \mathrm{m}$ on a cryostat (Leica Biosystems), with every fourth section throughout the entire HPC mounted on microscope slides. Sections were stained with cresyl violet and cover-slipped for quantification of HPC lesion volumes.

\section{Behavioral procedures: experiment 1}

For the contextual fear conditioning episode, rats $(n=96$ : $\mathrm{SHAM}=24$; $\mathrm{dHPC}=24 ; \mathrm{HPC}=28$ ) were transported individually from their home cage to the testing room in an opaque transport box and placed in the conditioning context. Following $180 \mathrm{~s}$ of exposure to the context four foot shocks $(0.9 \mathrm{~mA}, 2 \mathrm{~s}$ each) separated by an 88 -s intertrial interval were delivered (Zelikowsky et al., 2012). Following the final shock, rats remained in the context for $60 \mathrm{~s}$ before being returned to their home cage. Rats were brought back to the context $1,3,10$, or $30 \mathrm{~d}$ later for a single 8 min contextual fear memory test (no foot shocks). Each rat was conditioned once and tested once in the same context.

\section{Behavioral procedures: experiment 2}

The contextual fear conditioning episode and memory test were identical to experiment 1 for all rats $(n=50:$ SHAM $=25 ; \mathrm{HPC}=25)$. The context discrimination test consisted of one 8-min exposure in the novel context. The sequence of the tests was counter-balanced; half of the subjects in each group received the contextual fear memory test first, and half received the context discrimination test first (novel context exposure). The tests were separated by $6 \mathrm{~h}$, regardless of which occurred first. Testing took place 1,7 , or $15 \mathrm{~d}$ after conditioning.

\section{Behavioral procedures: experiment 3}

Rats (HPC, $n=5$; SHAM, $n=6$ ) received five consecutive days of MWT training, with eight swim trials each day for a total of 40 swim trials. The starting position for the first trial was randomly assigned to one of four cardinal points: N, S, E, or W. The sequence of start points for the eight swim trials varied each day. A trial began with placing a rat in the pool facing the pool wall at the assigned start position and ended either with the rat locating the platform and escaping, or until $60 \mathrm{~s}$ had elapsed. For trials with unsuccessful escapes, rats were led to the platform by the experimenter. Rats remained on the platform for $10 \mathrm{~s}$ after every trial before being placed back in the transport cage. Twenty-four hours following completion of MWT training, rats received one 60-s spatial memory probe trial. The probe followed the same general procedures as MWT training, except the escape platform was removed and all rats began the trial from a novel starting position (SE). Rats received the contextual fear conditioning episode $1 \mathrm{~d}$ after the MWT probe. Only the 15$\mathrm{d}$ training-to-test interval was used and all rats received the context discrimination test first, $6 \mathrm{~h}$ before the contextual fear memory test.

\section{Food restriction procedures: experiment 4}

Food restriction procedures were designed to ensure motivation at the time of visual discrimination training/testing rather than to drastically reduce bodyweight. Rats $(n=48)$ were given $\sim 4.0 \mathrm{~g}$ rat chow $/ 100 \mathrm{~g}$ bodyweight once daily until weights reached $\sim 95 \%$ of free-feeding values. The food reward (sweetened cereal; President's Choice Toasted Oat Os, Loblaw Companies Ltd, Canada) was introduced to rats in home cages during the same $3-\mathrm{d}$ period. Food restriction (18-25 g/d) continued throughout the experiment until endpoint, with bodyweights monitored daily and allowed to gradually increase by $\sim 5 \mathrm{~g}$ each week between training and testing. No sweetened cereal was given in home cages once training began. Rats received their daily allotment of chow between 5:30 and 7:30 P.M., which corresponded to 12-14 h before daily visual discrimination training sessions.

\section{Behavioral procedures: experiment 4}

Each rat $(n=48$ : SHAM $=24 ; \mathrm{HPC}=24)$ was habituated to the arena before commencement of visual discrimination training. Daily pretraining sessions lasted $10 \mathrm{~min}$ and were designed to gradually shape rats' behavior. On day 1, rats were free to explore the arena, objects and reward dishes. Food reward was available in the dishes and throughout the box. The objects were present, but not covering the food dishes. All rats explored the arena, reward dishes, objects, and consumed food reward. On day 2 , the reward was only available in the dishes and the objects were not covering the dishes. All rats consumed reward from both dishes. On the final day of pretraining, the reward was in the dishes (one piece in each dish) with the objects set on the dishes. When rats moved an object to access and consume a reward, the reward and object were replaced by the experimenter. All rats moved objects off the dishes repeatedly to access reward. All rats learned the general requirements of the task by the end of the third pretraining session (i.e., remove an object to receive a reward). 


\section{EX. 1}
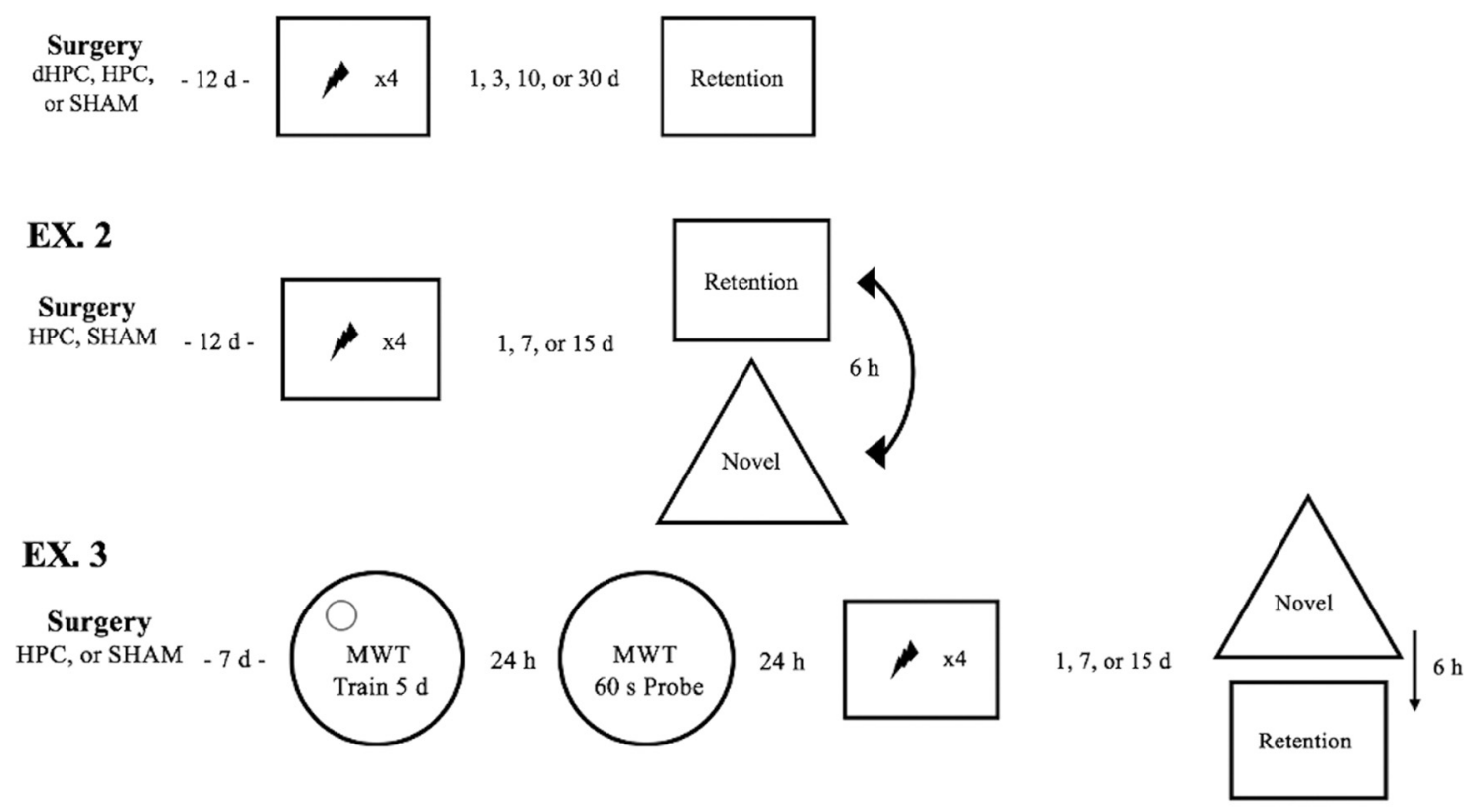

\section{EX. 4}

Surgery
HPC, SHAM $-12 d-$
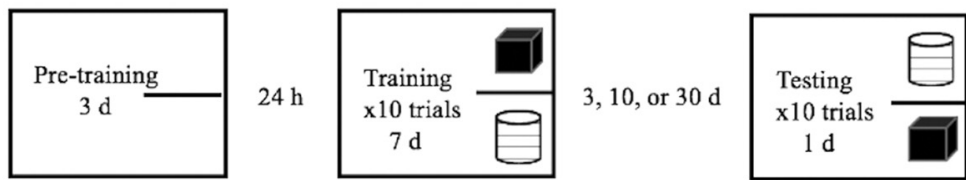

Figure 2. Experimental design and timeline. The figure illustrates the design of each experiment (experiments 1-4).

For training, rats received 10 trials a day until they achieved two consecutive days of $90 \%$ correct. Pairs of rats were brought to the testing room in a holding cage and received trials sequentially, in an alternating manner. Choices were recorded manually by the experimenter for each trial. The correct object $(\mathrm{S}+)$ and incorrect object (S-) were counterbalanced, so half of the HPC and SHAM rats learned each possible contingency (i.e., cylinder $\mathrm{S}+$, rectangle $\mathrm{S}-$; rectangle $\mathrm{S}+$, cylinder $\mathrm{S}-$ ). The position (dish A, or B) was varied pseudo-randomly, such that behavioral strategies (e.g., win/stay, lose/switch) could not facilitate the $90 \%$ correct criterion. When employing such strategies, the maximum correct\% using an $\mathrm{ABBABAABAB}$, or $\mathrm{BAABABBABA}$ design is $80 \%$ if a correct first choice is made and $70 \%$ if an incorrect first choice is made. The reward was not delivered until the correct object $(\mathrm{S}+)$ was displaced; therefore, rats could not simply locate the reward via olfaction and remove the object to access it. When a correct choice was made, the rat consumed the reward before being removed from the arena. When an incorrect choice was made, no reward was delivered and the rat was removed from the arena. Thus, a trial began with the rat being placed in the start position within the arena and ended once the correct object had been displaced and the reward was delivered and consumed, or the incorrect object had been displaced and no reward was delivered. Critically, the removable floor, walls, reward dishes, and objects were thoroughly cleaned with quat-sanitizer between every trial to further ensure rats could not use olfaction to resolve the discrimination. The intertrial interval was variable, but was 2 or $3 \mathrm{~min}$ because of cleaning procedures.

Rats were tested for memory retention 3,10 , or $30 \mathrm{~d}$ after the final training session. The procedures for retention testing were identical to those during training, with each rat receiving 10 trials on its previouslylearned object discrimination problem.

Experimental design and statistical analysis

Figure 2 illustrates the design of each experiment. Freezing was the index of contextual fear conditioning and memory expression (experiments 1-
3). All sessions were recorded through a high-definition USB camera connected to a PC laptop computer running FreezeFrame 4.0 software (Actimetrics, Coulbourn Instruments), which captured session video and freezing data. For conditioning sessions, freezing during the 30-s preceding each foot shock was analyzed (Zelikowsky et al., 2012; [time immobile (s)/30 (s) $\times 100=$ freezing\%]). For conditioning context and novel context testing, the entire session was analyzed ([time immobile (s)/ $480(\mathrm{~s}) \times 100=$ freezing\%]). Frame rate and motion detection thresholds were identical for all conditioning and testing sessions and employed FreezeFrame optimal system performance settings. Conditioning data were analyzed with a two-way ANOVA, with repeated measures (RM) for each foot shock delivery (group $\times$ trial) and Sidak's post hoc comparisons to detect any differences in learning rates between and within groups. Memory retention test and novel context data were analyzed with twoway ANOVA (group $\times$ retention interval) and Sidak's post hoc comparisons. Two-way RM ANOVA was used for each retention interval (1, 7, and $15 \mathrm{~d}$ ) with group and context as factors.

Context discrimination was calculated as a ratio [freezing\% (shock context)/freezing\% (shock context) + freezing\% (novel context) $\times$ $100=$ discrimination\%]. Discrimination values were then analyzed with one-sample $t$ tests with chance-level discrimination (i.e., 50\%) as the hypothesized value. For MWT training sessions, mean escape latency was analyzed with RM ANOVA by group (HPC, SHAM) $\times$ trial within training day, and group $\times$ day for the training period. For the probe trial, dwell time (s) in the correct quadrant ([time in quad 4/time in all quads]) was compared with average dwell time in incorrect quadrants ([mean time in incorrect quads/time in all quads]) for each rat then analyzed by group.

For visual discrimination, manually recorded training trial data were expressed as Correct $\%$ for each session (correct trials/total trials $\times 100$ ). Training scores were analyzed with a two-way ANOVA (group; SHAM, HPC) with RM (training day). Memory retention scores were analyzed with two-way ANOVA (group $\times$ retention interval). All statistical analyses performed in Prism 6. Behavioral data for all experiments are represented as mean \pm SEM. 

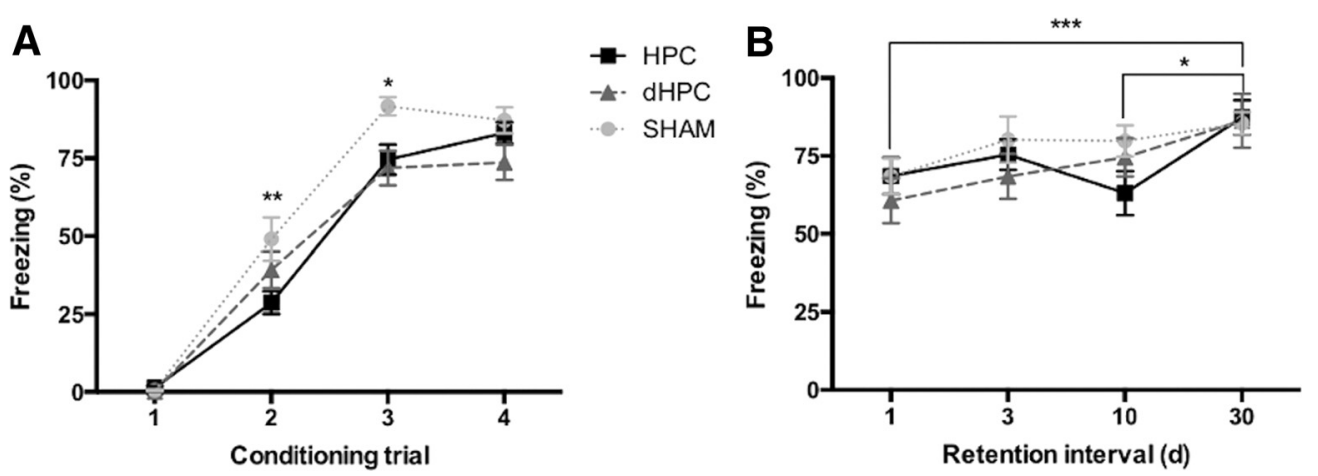

Figure 3. Contextual fear conditioning and retention tests. $A$, During acquisition, SHAM rats exhibited more freezing behavior than HPC rats on trial 2 (**), and HPC and dHPC rats on trial 3 (*). All groups displayed similar levels of freezing by the end of the conditioning episode. B, For retention tests, SHAM, dHPC, and HPC rats exhibited similar levels of freezing behavior at all retention intervals. On average, rats froze more during the $30-\mathrm{d}$ test than the $1-\mathrm{d}$ test $(* * *)$ and the $30-\mathrm{d}$ test versus $10-\mathrm{d}(*)$ test regardless of lesion condition. All data points expressed as mean \pm SEM.

The volume of intact HPC was quantified stereologically (Sparks et al., 2013) via the Cavalieri method using a brightfield microscope equipped with a motorized stage and StereoInvestigator software (MBF Bioscience). The measured volume of intact principal cell fields in each HPC-damaged brain was then divided by the mean HPC volume calculated from a subset of SHAM group animals $(n=6)$ and multiplied by 100 to yield intact HPC $\%$. Intact HPC\% was subtracted from 100 to yield HPC\% damage (100 $\left[\left(\mathrm{HPC} \mathrm{mm}^{3} /\right.\right.$ average $\left.\left._{(\mathrm{SHAM})} \mathrm{mm}^{3}\right) \times 100\right]=$ HPC\% damage $)$.

\section{Results}

Experiment 1: rats with hippocampal damage acquire a stable contextual fear memory and express it normally for at least $30 \mathrm{~d}$

Analysis of freezing during the conditioning episode yielded main effects of group, $F_{(2,76)}=5.412, p=0.0063$; trial, $F_{(3,228)}=297.4$, $p<0.0001$; and a group $\times$ trial interaction, $F_{(6,228)}=2.751, p=$ 0.0133. Post hoc comparisons found HPC rats froze less than SHAM rats on trials $2(p=0.0016)$ and $3(p=0.0101)$. dHPC rats froze less than SHAM rats on trial 3 ( $p=0.0043$; Fig. 3 ).

For the memory retention test, the groups did not differ $\left(F_{(2,67)}=0.9744, p=0.38\right)$, nor was the interaction between group and retention interval significant $\left(F_{(6,67)}=0.7617, p=0.6026\right)$. However, there was a main effect of retention interval $\left(F_{(3,67)}=\right.$ 5.34, $p=0.0019)$. Post hoc analysis confirmed differences in conditioned freezing between retention intervals ( 1 vs $30 \mathrm{~d}$, $p=0.0009$; 10 vs $30 \mathrm{~d}, p=0.031$; Fig. 3 ). In summary, all rats learned to fear the context during the conditioning episode and demonstrated memory for the experience at all retention intervals regardless of lesion condition.

Lesion volume estimates for dHPC group rats confirmed extensive and consistent damage across groups of animals tested at $1,3,10$, or $30 \mathrm{~d}$, as indicated by similar means $\left(F_{(3,20)}=0.5954, p=0.6254\right)$ and standard deviations (Table 2 ). A conservative proportion of $50 \%$ volume of the entire HPC was assigned to dHPC (Broadbent et al., 2004). Lesion estimates for HPC group rats indicated HPC damage ranged from substantial to extensive (65.11-89.34\%). Analysis of lesion volumes grouped by retention interval $(1,3,10$, and $30 \mathrm{~d})$ revealed a main effect of group $\left(F_{(3,27)}=6.03, p=\right.$ 0.028 ). Tukey's post hoc comparisons confirmed significant differences in HPC mean lesion volumes. The percent values are as follows: 1 versus $30 \mathrm{~d}=-7.836 \%$; three versus 10 $\mathrm{d}=8.501 \% ; 10$ versus $30 \mathrm{~d}=-10.40 \%$.

Because of the range of lesion volumes ( $\min =40.11 \%$; $\max =84.33 \%$ ), HPC damage versus freezing was analyzed with
Table 2. The table illustrates the minimum, mean, and maximum hippocampal damage values expressed in percent of the entire HPC for each experiment (SD; standard deviation)

\begin{tabular}{lllllc}
\hline Experiment & Lesion & Smallest & Mean & Largest & SD \\
\hline 1 & dHPC & $40.01 \%$ & $44.84 \%$ & $50.00 \%$ & $3.20 \%$ \\
& HPC & $65.11 \%$ & $73.31 \%$ & $84.33 \%$ & $4.86 \%$ \\
2 & HPC & $70.22 \%$ & $90.99 \%$ & $98.21 \%$ & $6.23 \%$ \\
3 & HPC & $79.41 \%$ & $87.04 \%$ & $92.72 \%$ & $5.62 \%$ \\
4 & HPC & $72.53 \%$ & $83.92 \%$ & $93.75 \%$ & $5.64 \%$ \\
\hline
\end{tabular}

Pearson correlation. For conditioning trials with significant effects of group, there was no significant correlation between magnitude of HPC damage and freezing (trial 2, $r=-0.2361, r^{2}=$ $0.05574, p=0.0827$; trial $3, r=-0.07587, r^{2}=0.005757, p=$ $0.5819, n=55)$. Similarly, correlation between HPC damage and freezing during the contextual fear retention tests was not statistically significant $\left(r=0.16, r^{2}=0.026, p=0.26, n=55\right)$. For retention tests, the coefficient of determination indicated that $2.6 \%$ of the variance in freezing was attributable to variation in extent of hippocampal damage. Table 2 illustrates hippocampal lesion volume data for every experiment.

\section{Experiment 2: rats with extensive to near-complete hippocampal damage exhibit normal levels of context discrimination for at least $15 \mathrm{~d}$}

For the conditioning episode, analysis of freezing by group $\times$ trial revealed main effects of group, $F_{(1,51)}=8.89, p=0.0033$; trial, $F_{(3,153)}=234.50, p<0.0001$; and a group $\times$ trial interaction, $F_{(3,153)}=3.57, p=0.016$. As in experiment 1 , post hoc comparisons confirmed between-group differences on trial 2 ( $p=$ $0.0021)$, and trial $3(p=0.0066)$, with HPC rats freezing less than SHAMs (Fig. 4).

Freezing in the shock context during retention tests was similar for subgroups of HPC and SHAM rats tested at different intervals. There was no effect of group, $F_{(1,47)}=0.87$, $p=0.35$; nor retention interval, $F_{(2,47)}=1.02, p=0.37$. There was a group $\times$ retention interval interaction, $F_{(2,47)}=3.25$, $p=0.048$; however, post hoc comparisons did not indicate statistical differences between HPC and SHAM subgroups freezing at any retention interval $(1 \mathrm{~d}, p=0.1845 ; 7 \mathrm{~d}$, $p=0.5957$; $15 \mathrm{~d}, p=0.3555$; Fig. 4).

Analysis of freezing in the novel context yielded an effect of retention interval, $F_{(2,47)}=8.61, p=0.0007$; and a group $\times$ retention interval interaction, $F_{(2,47)}=3.39, p=0.042$. Post hoc 
A

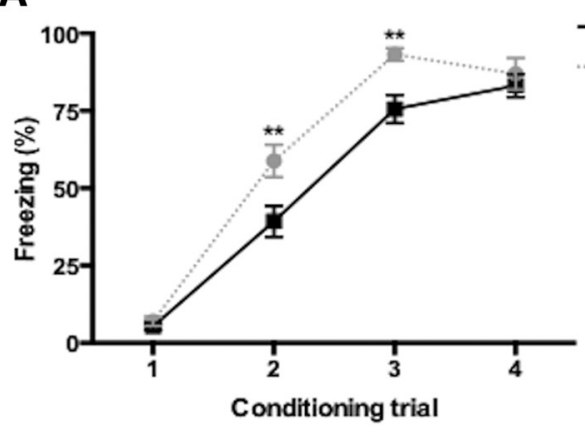

B

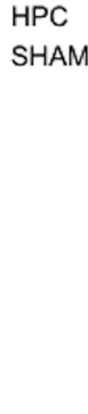

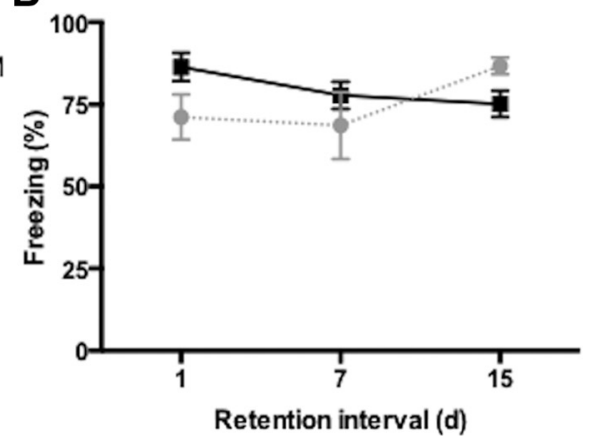

Figure 4. Contextual fear conditioning and memory tests. $\boldsymbol{A}$, During the conditioning episode, HPC rats exhibited less freezing than SHAM rats on trials 2 and $3 . \boldsymbol{B}$, HPC and SHAM rats performed similarly on the context memory test at 1-, 7-, and 15-d retention intervals. Statistically significant between-group differences are indicated (**). All data points expressed as mean \pm SEM.
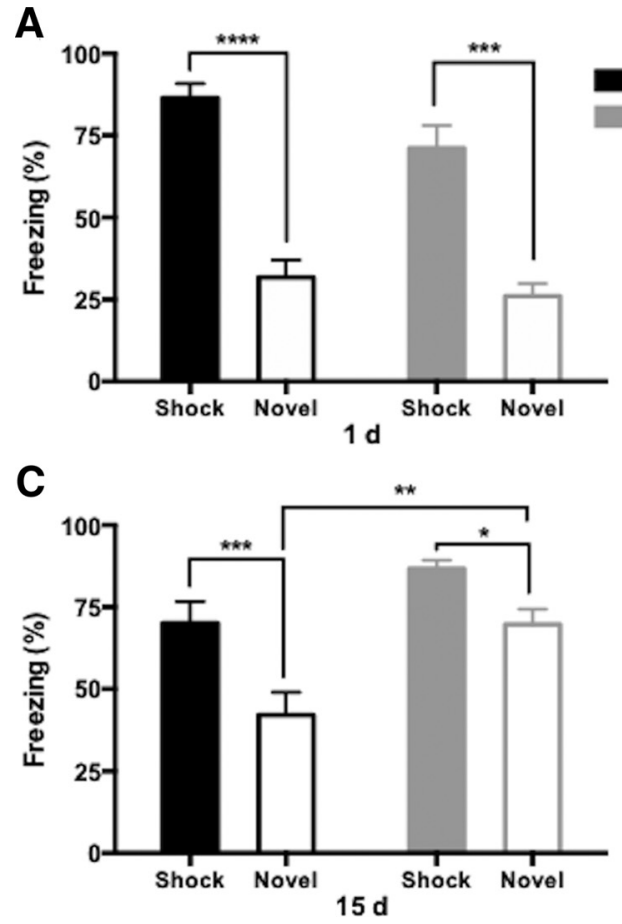

B
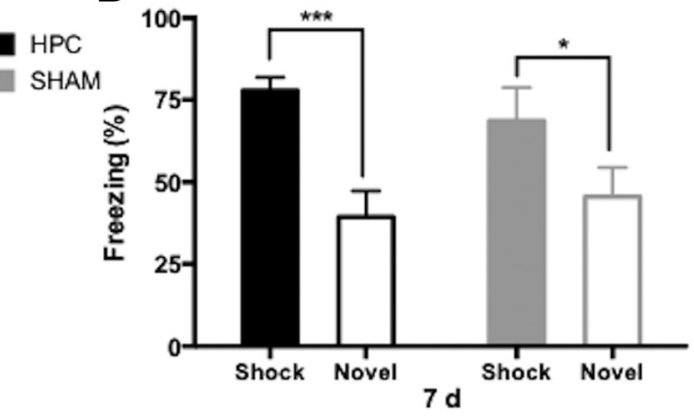

D

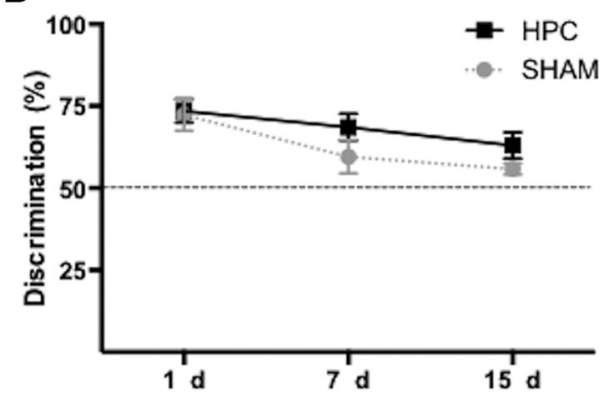

Figure 5. Context discrimination over time. Rats tested $(\boldsymbol{A}) 1 \mathrm{~d},(\boldsymbol{B}) 7 \mathrm{~d},(\boldsymbol{C}) 15 \mathrm{~d}$ after contextual fear conditioning exhibited accurate context discrimination (within-group differences, asterisks and narrow connector lines). Compared with HPC rats, SHAM rats froze more in the novel context during the 15-d test (between-group difference wide connector lines $* *$ ). $\boldsymbol{D}$, Rats demonstrated above chance context discrimination at all train-to-test intervals, except for the SHAM group on the 7-d test. Dashed line denotes chance-level discrimination. All data points expressed as mean \pm SEM.

comparisons identified a statistical difference on the 15-d test, with SHAM rats freezing more in the novel context than did HPC rats; $p=0.0103$. Analysis of freezing for each retention interval with group and context as factors yielded an effect of context on the 1-, 7-, and 15-d tests $\left(F_{(1,15)}=62.84, p<0.0001\right.$; $F_{(1,15)}=33.58, p<0.0001 ; F_{(1,17)}=23.16, p=0.0002$, respectively) and an effect of group on the 1 - and 15 -d tests $\left(F_{(1,15)}=8.250, p=\right.$ $0.0116 ; F_{(1,17)}=11.66, p=0.0033$, respectively; Fig. 5).

Discrimination ratio calculations and subsequent analysis yielded a main effect of retention interval, $F_{(2,47)}=6.00, p=$ 0.0048 ; no effect of group, $F_{(1,47)}=3.176, p=0.0812$; and no interaction, $F_{(2,47)}=0.5168, p=0.5998$. One-sample $t$ tests indicated above chance (i.e., 50\%) context discrimination by HPC group rats on 1-, 7-, and 15-d tests $(p=0.0002 ; p=0.002$; $p=0.01$, respectively). The SHAM group discrimination was above chance on 1-, 15-, but not 7 -d tests $(p=0.003 ; p=0.009$; $p=0.10$, respectively; Fig. 5). Analysis of lesion volumes revealed similar means between subgroups of rats tested at 1-, 7-, and 15d retention intervals (ANOVA: $p=0.56$ ).

Experiment 3: behavioral verification of complete hippocampal dysfunction and intact context discrimination following extensive HPC lesions

Over $5 \mathrm{~d}$ of MWT training, escape latencies decreased. Analysis of mean escape latency by group (SHAM, HPC) across training days revealed main effects of group; $F_{(1,9)}=11.36, p=0.008$, and day; $F_{(4,36)}=52.47, p<0.0001$. Post hoc comparisons indicated HPC escape latencies were longer than SHAM latencies on training days 2 and $3 ; p=0.0021 ; p=0.045$, respectively (Fig. 6). For the probe test, dwell time in the correct quadrant was greater than in incorrect quadrants for SHAM rats; $p=0.001$, but not HPC rats; $p>0.99$, which performed at chance levels (Fig. 6). 
A

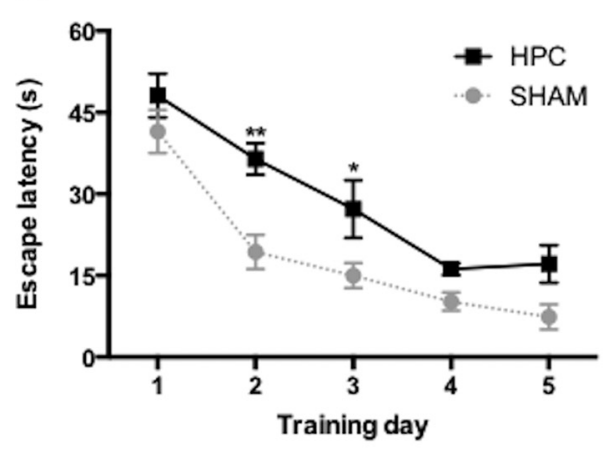

C

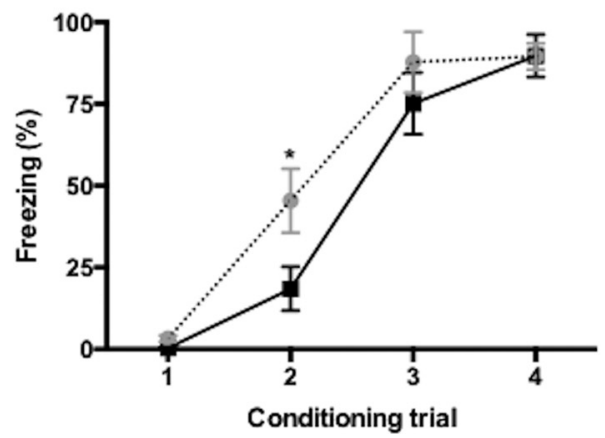

B

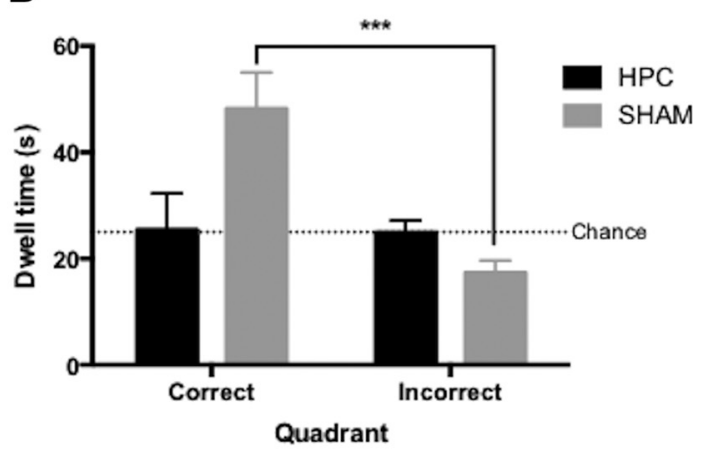

D

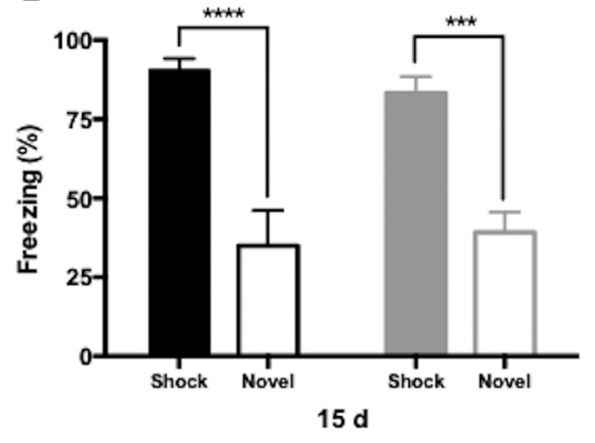

Figure 6. MWT, contextual fear conditioning, and context discrimination data from the behavioral verification of lesion experiment. $\boldsymbol{A}$, SHAM and HPC rats decreased MWT escape latencies over five training days (between-group differences denoted, *** and *). B, SHAM rats demonstrated memory for the platform location on the 60-s spatial probe (***), but HPC rats did not as indicated by equal dwell time across quadrants. C, During the conditioning episode HPC rats froze less than SHAM rats on trial 2 (*). D, Both groups expressed accurate context discrimination by freezing more in the shock than novel context on the 15-d test (HPC ****; SHAM ***). All data points expressed as mean \pm SEM.

Analysis of freezing by group $\times$ trial for the conditioning episode revealed main effects of group, $F_{(1,36)}=71.73, p<0.0001$; and trial; $F_{(1,36)}=4.61, p=0.039$. Post hoc comparisons revealed a between-group difference on trial $2(p=0.037)$, with HPC rats exhibiting less freezing compared with SHAMs (Fig. 6). For the 15$\mathrm{d}$ memory and discrimination test, there was a main effect of context $F_{(1,9)}=19.75, p<0.0001$; and no effect of group, $F_{(1,9)}=$ $0.02,765, p=0.8716$; with post hoc comparisons confirming both groups froze more in the conditioning context than novel context (SHAM, $p=0.0002$; HPC, $p<0.0001$; Fig. 6). Damage to the HPC ranged from extensive $(79.41 \%)$ to near-complete $(92.72 \%)$, and was consistent between rats (mean $=87.04 \%$; $\mathrm{SD}=5.62 \% ; n=5$ ).

\section{Experiment 4: rats with hippocampal damage maintain and express accurate object discrimination memory for up to $30 \mathrm{~d}$}

Analysis of number of correct trials revealed a main effect of day, $F_{(6,276)}=364.20, p<0.0001$; no effect of group $F_{(1,46)}=0.1706$, $p=0.6815$; and no significant interaction $F_{(6,276)}=1.406, p=$ 0.2123 . Analysis of retention tests yielded a main effect of retention interval $\left(F_{(2,42)}=4.054, p=0.025\right)$. Like the learning data, neither an effect of group, nor an interaction was present $\left(_{(1,42)}=0.1386, p=0.7115 ; F_{(2,42)}=0.6584, p=0.5229\right.$, respectively). Post hoc comparisons (Tukey) between retention intervals indicated a significant difference in mean correct percentage between the 3- and 30-d tests ( $p=0.0241$; Fig. 7$)$. A tally of firsttrial scores resulted in the following: 8/8 SHAM and 7/8 HPC rats chose the $\mathrm{S}+$ object on day 3; 6/8 SHAM and 7/8 HPC on day 10; and 6/8 SHAM and HPC chose correctly on the $30-\mathrm{d}$ test. Analysis of HPC \% damage indicated similar means between retention intervals (ANOVA: $p=0.36$ ). Figure 8 depicts representative examples of minimum, mean, and maximum lesions for all experiments.

\section{Discussion}

In four experiments, we examined the maintenance and behavioral expression of long-term memories acquired in the absence of the HPC. The hypothesis that the HPC is necessary for stable and detailed long-term memories was tested. Neither partial (dHPC) nor extensive (HPC) damage impaired the remote expression of contextual fear memory. No sign of memory decay was observed for up to $30 \mathrm{~d}$. In addition, in two experiments extensive to near-complete hippocampal damage made before learning did not affect the longevity of context memory or accuracy of context discrimination for up to $15 \mathrm{~d}$ after learning. The remote (30 d) expression of a two-choice object discrimination was also found to be normal if acquired in the absence of the HPC. Critically, testing in the spatial cue version of the MWT, a task that is relatively insensitive to partial damage to the HPC (Moser et al., 1995), verified that lesion procedures resulted in a large functional impairment. This greatly reduces the likelihood that the results were confounded by spared hippocampal function. Collectively, these findings demonstrate that non-hippocampal networks can acquire, maintain, and express memories, scoring against the hypothesis that important properties of context memories and discrimination require the HPC and its systems consolidation mechanisms (Zelikowsky et al., 2012).

The discovery that context memories formed in the absence of HPC damage exhibit normal longevity is at odds with the only other study to investigate this question. Despite employing Zelikowsky et al. (2012)'s experimental procedures as described, we observed no memory decay in dHPC or HPC rats, regardless of magnitude of damage. One small possibility 
A

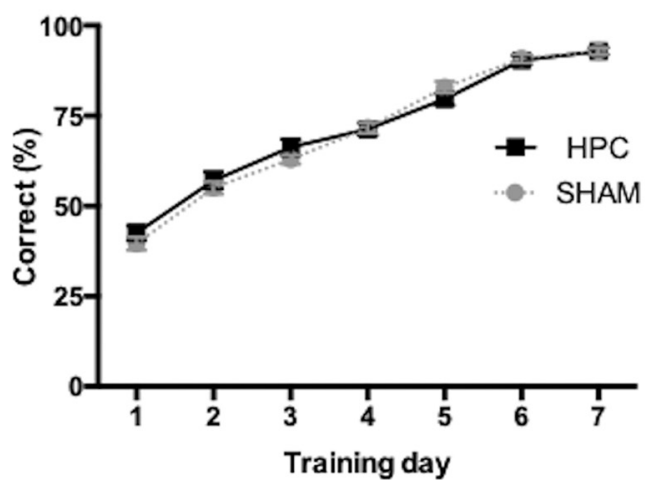

B

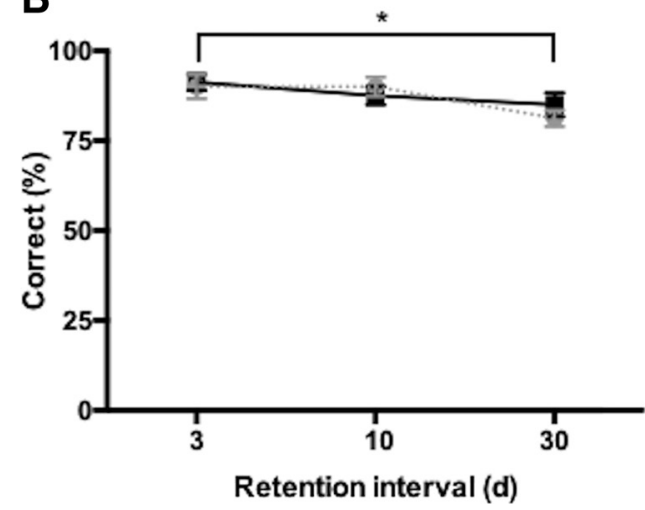

Figure 7. Object discrimination training and memory retention tests. $A, H P C$ and SHAM groups learned to discriminate $S+$ from $S-$ to $>90 \%$ accuracy by days 6 and 7 . $B$, Memory performance of HPC and SHAM groups was similar at each train-to-test interval. There was a statistically significant decrease in memory performance exhibited by both groups between the 3- and 30$\mathrm{d}$ tests ( $* ; 3$ vs $30 \mathrm{~d}$ : HPC, $-6.26 \%$; SHAM, $-8.75 \%$ ). All data points expressed as mean \pm SEM.

Ex 1. $-3.0$

$-4.4$
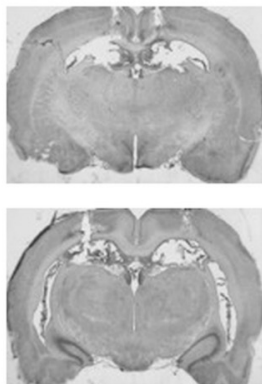

$-5.8$

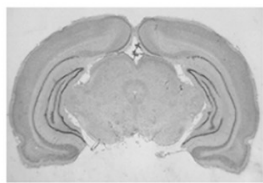

dHPC: $45.1 \%$

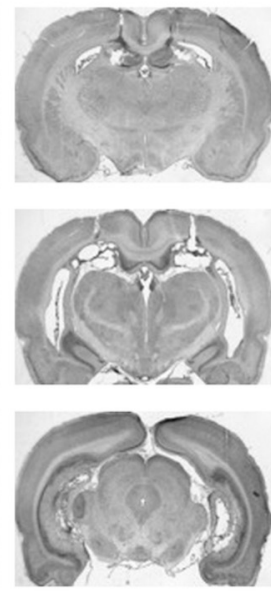

HPC: $73.31 \%$
Ex 2.
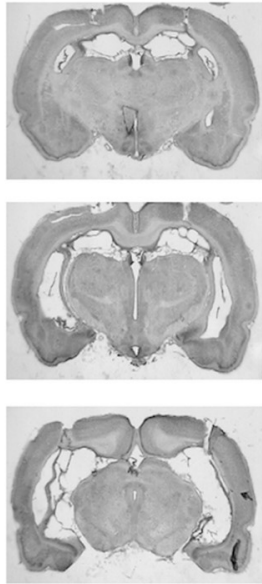

HPC: $90.99 \%$
Ex 3 .
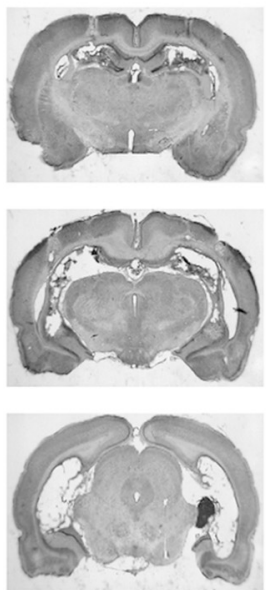

HPC: $87.04 \%$
Ex 4.

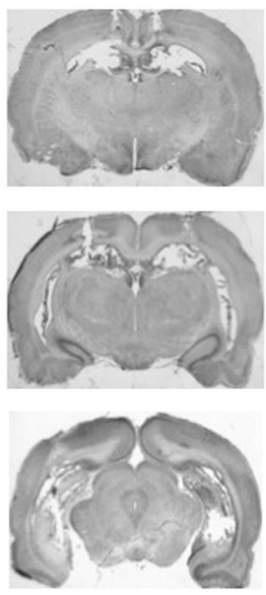

HPC: $83.92 \%$

Figure 8. HPC histology. Images of coronal sections taken at approximately $-3.0,-4.4$, and $-5.8 \mathrm{~mm}$ posterior to bregma illustrating approximate mean damage to the HPC for all four experiments.

is that the addition of an uncontrolled dose trimethoprim sulfa (an antibiotic/protein synthesis inhibitor) to rats' drinking water for a week during the surgical recovery period affected memory performance. Anterograde memory impairments during sulfa drug regimens have been reported by human patients (Sternbach and State, 1997). It is unknown whether this side effect extends to rodents, or whether this antibiotic could cause rapid decay of memory selectively in rats with dHPC damage. In our view, the uncontrolled use of trimethoprim sulfa is a potential confound. We omitted the antibiotic because we could not replicate the dosage, which was not reported. Therefore, it could not be held constant as an independent variable. Because the prolonged use of antibiotic and the observation of rapid memory decay in dHPC damaged rats are unique to Zelikowsky et al. (2012), drawing conclusions about the findings may not be possible. In contrast, we observed no retention impairments across three separate experiments on 1-, 3-, 7-, 10-, 15-, or 30-d tests. Our findings are consistent with one report of normal performance on a 14-d contextual fear memory test by rats with complete hippocampal damage. This study employed a mixed, retrograde/anterograde testing design in which rats underwent two rounds of conditioning, one before HPC damage and one following damage (Wiltgen et al., 2006). We chose an exclusively anterograde design to eliminate the possibility of memory savings from the prelesion conditioning episode. Further study is needed to determine whether context memories acquired in the absence of the HPC persist beyond $30 \mathrm{~d}$ and whether antibiotics selectively disrupt memory consolidation in rats with HPC damage compared with intact rats.

Intact rodents exhibit a context-specific fear response shortly after a fear conditioning episode, but over time freezing behavior transfers to unconditioned contexts. This generalization thought to reflect a loss of memory detail, and a shift from HPC-dependent to HPC-independent memory retrieval (Wiltgen and Tanaka, 2013). Whereas previous lesion studies involving rats with partial HPC damage reported impaired retention for context discrimination, we found that rats with extensive HPC damage exhibited normal context discrimination on 1-, 7-, and 15-d tests. Importantly, the finding of intact discrimination by HPC rats on the 15-d test was replicated in a follow-up experiment 
which also verified that our lesion procedures severely disrupted HPC function. Although the context discrimination we used was low-to-medium with regard to cue overlap and ambiguity, intact rats did not outperform HPC-damaged rats. These data oppose the idea that non-hippocampal networks only support a fragile, contextually-impoverished version of long-term memory (Winocur et al., 2013) and add to examples of intact context discrimination in the absence of the HPC in rodents.

Wang et al. (2009) conditioned groups of mice using a discriminative fear to context procedure, then performed HPC lesions 1 or $42 \mathrm{~d}$ after learning. Neither retrograde amnesia for the conditioning episode, nor impaired context discrimination was reported. The extent of hippocampal lesions was described as near complete (Wang et al., 2009). The authors concluded that the HPC is not always required for the long-term expression of detailed context memories. In our view, repeated experience in the paired and unpaired contexts before HPC damage likely yielded detailed memories for each context in non-hippocampal networks. In line with this, we previously found that rats with HPC lesions made after distributed fear conditioning episodes exhibited normal discrimination when later tested in the conditioning context and a novel context (Lehmann et al., 2009). More recently, Lee et al. (2017) found that rats with $\sim 80 \%$ HPC lesions made before discriminative fear conditioning exhibited normal context preference $3 \mathrm{~d}$ after learning. Importantly, separate groups of HPC-damaged rats displayed normal context preference behavior even under very weak conditioning parameters (Lee et al., 2017). However, HPC lesions made after conditioning resulted in retrograde amnesia and impaired discrimination. These findings provide evidence that extensive damage to the HPC does not invariably result in impaired maintenance and expression of long-term contextual fear memories, or context discriminations. By extension, the consolidation of these memories does not appear to be uniquely dependent on the HPC.

Although rats with and without HPC damage learn visual discriminations, some data suggest these memories decay when they are formed in the absence of the HPC (Vnek and Rothblat, 1996; Wiig et al., 1996; Broadbent et al., 2007). In our fourth experiment, we sought to clarify whether the HPC is required for the longevity of visual discrimination memories. We found that rats with extensive pretraining HPC damage ( $\sim 83 \%$ average) maintained and expressed object discrimination memories as well as SHAM rats when tested 3, 10, or $30 \mathrm{~d}$ after learning. All rats exhibited a small decrement in performance on the 30-d test indicative of minor memory decay. To our knowledge, a 30-d retention interval is the longest to date for visual discrimination memories in rats, and this experimental design was unique. Previous studies typically used some combination of extended training and testing on multiple discrimination problems, posttraining lesions and testing, then training on new problems, and/ or incomplete HPC disruption procedures. We suggest these design elements increase the probability of confounding relearning with memory performance and incomplete HPC damage with the complete loss of HPC function. In contrast, it is unlikely that discrimination performance was because of relearning rather than memory expression in our study, as retention testing consisted of 10 trials and the majority (75\%) of SHAM and HPC rats chose correctly on the first trial during the 30-d retention test. In addition, HPC lesions were systematically quantified and similar extensive damage was sufficient to cause profound impairments in the MWT (experiment 3). These findings indicate that visual discrimination memories exhibit normal longevity when acquired in the absence of the HPC.
Several lines of research have shown that regions outside the HPC are important for normal context memories, memory longevity and specificity, and cognitive control of discriminative behaviors. These include the retrosplenial cortex, medial prefrontal cortex, striatum, amygdala, and nucleus reuniens (Cowansage et al., 2014; Nelson et al., 2014; Kitamura et al., 2017; Ramanathan et al., 2018). This network normally includes the HPC and exhibits bidirectional monosynaptic and polysynaptic connectivity, but also HPC-independent interconnectivity and projections to behavioral effector regions (McDonald, 1998; McKenna and Vertes, 2004; Hoover and Vertes, 2007; Gruber and McDonald, 2012). The data we present here provide evidence that this network can support a version of long-term memories that are neither fragile, nor bereft of detail when the HPC is damaged before learning. By extension, the representational complexity of non-HPC memories may be greater than purported by systems consolidation-based theories.

\section{References}

Anagnostaras SG, Maren S, Fanselow MS (1999) Temporally graded retrograde amnesia of contextual fear after hippocampal damage in rats: within-subjects examination. J Neurosci 19:1106-1114.

Antoniadis EA, McDonald RJ (2000) Amygdala, hippocampus and discriminative fear conditioning to context. Behav Brain Res 108:1-19.

Broadbent NJ, Clark RE (2013) Remote context fear conditioning remains hippocampus-dependent irrespective of training protocol, training-surgery interval, lesion size, and lesion method. Neurobiol Learn Mem 106:300-308.

Broadbent NJ, Squire LR, Clark RE (2004). Spatial memory, recognition memory, and the hippocampus. Proc Natl Acad Sci USA 101:1451514520 .

Broadbent NJ, Squire LR, Clark RE (2007) Rats depend on habit memory for discrimination learning and retention. Learn Mem 14:145-151.

Cowansage KK, Shuman T, Dillingham BC, Chang A, Golshani P, Mayford M (2014) Direct reactivation of a coherent neocortical memory of context. Neuron 84:432-441.

Driscoll I, Howard SR, Prusky GT, Rudy JW, Sutherland RJ (2005) Seahorse wins all races: hippocampus participates in both linear and non-linear visual discrimination learning. Behav Brain Res 164:29-35.

Epp J, Keith JR, Spanswick SC, Stone JC, Prusky GT, Sutherland RJ (2008) Retrograde amnesia for visual memories after hippocampal damage in rats. Learn Mem 15:214-221.

Frankland PW, Filipkowski RK, Cestari V, McDonald RJ, Silva AJ (1998) The dorsal hippocampus is essential for context discrimination but not for contextual conditioning. Behav Neurosci 112:863-874.

Gidyk DC, Deibel SH, Hong NS, McDonald RJ (2015) Barriers to developing a valid rodent model of Alzheimer's disease: from behavioural analysis to etiological mechanisms. Front Neurosci 9:245.

Gruber AJ, McDonald RJ (2012) Context, emotion, and the strategic pursuit of goals: interactions among multiple brain systems controlling motivated behavior. Front Behav Neurosci 6:50.

Hoover WB, Vertes RP (2007) Anatomical analysis of afferent projections to the medial prefrontal cortex in the rat. Brain Struct Funct 212:149-179.

Kim J, Fanselow M (1992) Modality-specific retrograde amnesia of fear. Science 256:675-677.

Kitamura T, Ogawa SK, Roy DS, Okuyama T, Morrissey MD, Smith LM, Redondo RL, Tonegawa S (2017) Engrams and circuits crucial for systems consolidation of a memory. Science 356:73-78.

Lee JQ, Zelinski EL, McDonald RJ, Sutherland RJ (2016) Heterarchic reinstatement of long-term memory: a concept on hippocampal amnesia in rodent memory research. Neurosci Biobehav Rev 71:154-166.

Lee JQ, Sutherland RJ, McDonald RJ (2017) Hippocampal damage causes retrograde but not anterograde memory loss for context fear discrimination in rats. Hippocampus 27:951-958.

Lehmann H, Lacanilao S, Sutherland RJ (2007) Complete or partial hippocampal damage produces equivalent retrograde amnesia for remote contextual fear memories. Eur J Neurosci 25:1278-1286. 
Lehmann H, Sparks FT, Spanswick SC, Hadikin C, McDonald RJ, Sutherland RJ (2009) Making context memories independent of the hippocampus. Learn Mem 16:417-420.

Lehmann H, Rourke BK, Booker A, Glenn MJ (2013) Single session contextual fear conditioning remains dependent on the hippocampus despite an increase in the number of context-shock pairings during learning. Neurobiol Learn Mem 106:294-299.

Maren S, Aharonov G, Fanselow MS (1997) Neurotoxic lesions of the dorsal hippocampus and Pavlovian fear conditioning in rats. Behav Brain Res $88: 261-274$

McDonald AJ (1998) Cortical pathways to the mammalian amygdala. Prog Neurobiol 55:257-332.

McDonald RJ, Hong NS (2013) How does a specific learning and memory system in the mammalian brain gain control of behavior? Hippocampus 23:1084-1102.

McDonald RJ, King AL, Wasiak TD, Zelinski EL, Hong NS (2007) A complex associative structure formed in the mammalian brain during acquisition of a simple visual discrimination task: dorsolateral striatum, amygdala, and hippocampus. Hippocampus 17:759-774.

McKenna JT, Vertes RP (2004) Afferent projections to nucleus reuniens of the thalamus. J Comp Neurol 480:115-142.

Moser MB, Moser EI, Forrest E, Andersen P, Morris RG (1995) Spatial learning with a minislab in the dorsal hippocampus. Proc Natl Acad Sci USA 92:9697-9701.

Mumby DG, Astur RS, Weisend MP, Sutherland RJ (1999) Retrograde amnesia and selective damage to the hippocampal formation: memory for places and object discriminations. Behavioural Brain Res 106:97-107.

Nelson AJD, Hindley EL, Haddon JE, Vann SD, Aggleton JP (2014) A novel role for the rat retrosplenial cortex in cognitive control. Learn Mem 21:90-97.

Ramanathan KR, Jin J, Giustino TF, Payne MR, Maren S (2018) Prefrontal projections to the thalamic nucleus reuniens mediate fear extinction. Nat Commun 9:4527.

Scott GA, Saucier DM, Lehmann H (2016) Contrasting the amnesic effects of lesions and temporary inactivations of the hippocampus on context and spatial memory. J Behav Brain Sci 6:1-27.

Sekeres MJ, Moscovitch M, Winocur G (2017) Mechanisms of memory consolidation and transformation. In: Studies in neuroscience, psychology and behavioral economics. Cognitive neuroscience of memory consolidation, pp 17-44. San Diego: Springer International Publishing.

Sparks FT, Spanswick SC, Lehmann H, Sutherland RJ (2013) Neither time nor number of context-shock pairings affect long-term dependence of memory on hippocampus. Neurobiol Learn Mem 106:309-323.

Sternbach H, State R (1997) Antibiotics: neuropsychiatric effects and psychotropic interactions. Harv Rev Psychiatry 5:214-226.

Sutherland RJ, Weisend MP, Mumby D, Astur RS, Hanlon FM, Koerner A, Thomas MJ, Wu Y, Moses SN, Cole C, Hamilton DA, Hoesing JM (2001) Retrograde amnesia after hippocampal damage: recent vs. remote memories in two tasks. Hippocampus 11:27-42.

Sutherland RJ, O'Brien J, Lehmann H (2008) Absence of systems consolidation of fear memories after dorsal, ventral, or complete hippocampal damage. Hippocampus 18:710-718.

Vnek N, Rothblat LA (1996) The hippocampus and long-term object memory in the rat. J Neurosci 16:2780-2787.

Wang SH, Teixeira CM, Wheeler AL, Frankland PW (2009) The precision of remote context memories does not require the hippocampus. Nat Neurosci 12:253-255.

Wible CG, Shiber JR, Olton DS (1992) Hippocampus, fimbria-fornix, amygdala, and memory: object discriminations in rats. Behav Neurosci 106:751-761.

Wiig KA, Cooper LN, Bear MF (1996) Temporally graded retrograde amnesia following separate and combined lesions of the perirhinal cortex and fornix in the rat. Learn Mem 3:313-325.

Wiltgen BJ, Sanders MJ, Anagnostaras SG, Sage JR, Fanselow MS (2006) Context fear learning in the absence of the hippocampus. J Neurosci 26:5484-5491.

Wiltgen BJ, Tanaka KZ (2013) Systems consolidation and the content of memory. Neurobiol Learn Mem 106:365-371.

Winocur G, Moscovitch M, Sekeres MJ (2013) Factors affecting graded and ungraded memory loss following hippocampal lesions. Neurobiol Learn Mem 106:351-364.

Zelikowsky M, Bissiere S, Fanselow MS (2012) Contextual fear memories formed in the absence of the dorsal hippocampus decay across time. J Neurosci 32:3393-3397. 